\title{
Group theoretical analysis of a system of monopoles
}

\author{
S.C. Joshi* , V.P. Pandey and B.S. Rajput \\ High Energy Physics, Department of Physics, Kumaun University \\ Nainital - 263 002, India
}

\begin{abstract}
Investigating the behavior of a monopole moving in the field of another monopole we have obtained energy eigenvalue and eigenfunctions of the system. It has been demonstrated that isotropic harmonic oscillator is $(n+1)(n+2) / 2$ fold degenerate and $\mathrm{SU}(3)$ is the algebra to describe this system. The energy eigenvalue of monopolonium is modified from the usual energy eigenvalue of hydrogen atom due to the magnetic charge and Bohr radius of the system is very small in comparison to atomic Bohr radius. It has also been demonstrated that Hamiltonian of the monopolonium is invariant under $\mathrm{O}(4)$ and degree of degeneracy is $n^{2}$ fold.
\end{abstract}

\section{INTRODUCTION}

Physicists were fascinated by magnetic monopole since its ingenious idea was put forward by Dirac ${ }^{(1,2)}$ showing that mere existence of magnetic charge implies the quantization of electric charge. Ever since Dirac wrote down the quantization condition for magnetic charge, there have been many difficulties ${ }^{(1,2,3)}$ encountered in the scattering of magnetic monopole. The theory of magnetic monopole since its inception went through, two significant advancements, the quantum field theory of interacting electric and magnetic charges as developed by Schwinger ${ }^{(4)}$ and the local Lagrangian quantum field theoretic formulation developed by Zwanziger ${ }^{(5)}$. In his approach Schwinger postulated both the Hamiltonian and the commutation rules, which while successful, seems an adhoc method of formulating a quantum field theory. The basic variables in his theory, were the two transverse potentials $\overrightarrow{\mathrm{A}}^{\mathrm{T}}$ and $\overrightarrow{\mathrm{B}}^{\mathrm{T}}$ which were not canonically conjugate. Zwanziger's formulation begins with a Lagrangian, but the price he pays for

\footnotetext{
${ }^{*}$ Corresponding author
} 
locality is the doubling of the number of photon variables. As such the canonical and physical structure of his theory requires a great deal of clarifications. We have under taken $^{(6,7,8)}$ the study of scattering of a photon and a magnetic monopole and it has been shown that photon responsible for compton scattering of magnetic monopole is highly energetic. We have also undertaken the study of S-matrix expansion and deduced Feynmann rules for different charge particle and photon interactions ${ }^{(6)}$. We have further studied the Pauli equation for monopolonium and it has been demonstrated that monopolonium is very strongly bounded and energy of the system is very high in comparison to bound states of quantum electrodynamics. We have also undertaken the study of anti-monopolium ${ }^{(8)}$ (i.e. bound state of monopole and anti monopole ) and have demonstrated that this state is very short lived and decays into two or three highly energetic photons depending on the spin- statistics of the particles involved. Extending this work in the present paper we have undertaken the study of magnetic monopole in group theoretical framework.

\section{ENERGY SPECTRUM AND DEGENERACIES ASSOCIATED WITH HARMONIC OSCILLATOR :}

In order to study the degeneracy of harmonic oscillator we start with the following Hamiltonian

$$
\hat{\mathrm{H}}=\frac{\hat{\pi}^{2}}{2 \mathrm{~m}}+\frac{\mathrm{mr}^{2} \omega^{2}}{2}
$$

where $\vec{\pi}$ is the gauge invariant linear momentum operator defined as ${ }^{(6)}$

$$
\vec{\pi}=(\overrightarrow{\mathrm{P}}-\mathrm{gB})
$$

where $g$ is the charge associated with magnetic monopole $\vec{V}$ is the spatial component of magnetic four potential $\left\{\mathrm{B}^{\mu}\right\}$. The Schrocdinger equation for this system may be written as

$$
\hat{\mathrm{H}} \psi=\mathrm{E} \psi
$$

where $\hat{\mathrm{H}}$ is given by equation (1). Separating equation (3) into radial and angular parts by defining ${ }^{(6)}$

$$
\psi=\mathrm{R}(\mathrm{r}) \mathrm{Y}_{\mathrm{g}, \mathrm{l}, \mathrm{m}}(\theta, \phi)
$$


where $\mathrm{Y}_{\mathrm{g}, \mathrm{l}, \mathrm{m}}(\theta, \phi)$ are monopole harmonics we get the following radial equation

$$
\frac{\mathrm{d}^{2} \mathrm{R}}{\mathrm{dr} \mathrm{r}^{2}}+\frac{2}{\mathrm{R}} \frac{\mathrm{dR}}{\mathrm{dr}}+\left[2 \mathrm{~m}\left\{\mathrm{E}-\mathrm{mr}^{2} \omega^{2}\right\}-\frac{\mathrm{l}(\mathrm{l}+1)}{\mathrm{r}^{2}}\right] \mathrm{R}=0
$$

\section{Introduction to the dimensionless quantities}

$$
\xi=\sqrt{\mathrm{ar}}=\sqrt{\frac{\mathrm{m} \omega}{\hbar}} \mathrm{r}
$$

and $\quad \xi=\mathrm{E} / \hbar \omega$

equation (5) gets the following form

$$
\frac{\mathrm{d}^{2} \mathrm{R}}{\mathrm{d} \xi^{2}}+\left[2 \xi-\xi^{2}-\frac{\mathrm{l}(\mathrm{l}+1)}{\xi^{2}}\right] \mathrm{R}(\xi)=0
$$

Substituting

$$
\begin{aligned}
& \xi=2\left(\mathrm{n}+\mathrm{q}+\frac{1}{4}\right) \\
& \mathrm{l}(\mathrm{l}+1)=4 \mathrm{q}\left(\mathrm{q}-\frac{1}{2}\right)
\end{aligned}
$$

introducing the variables

$$
\mathrm{P}=\xi^{2}=\mathrm{dr}^{2}
$$

and writing a function

$$
w(\rho)=e^{-\rho / 2} \rho^{-q} R(r)
$$

equation (5) may be written as

$$
\frac{\rho d^{2} w(\rho)}{d \rho^{2}}+\left(2 q+\frac{1}{2}-\rho\right) \frac{d w(\rho)}{d \rho}+n w(\rho)=0
$$

which has the form of confluent hypergeometric differential equation. Solution of this equation may be written as 


$$
\mathrm{W}(\rho)=\mathrm{F}\left(-\mathrm{n}, 2 \mathrm{q}+\frac{1}{2} ; \rho\right)
$$

which is acceptable solution (in the form of a polynomial) for $n=0,1,2 \ldots$. . Furthermore, equation (8) gives

$$
\mathrm{q}=\frac{1}{2}(1+1)
$$

Substituting this value into equation (8) we get

$$
\varepsilon=2 \mathrm{n}+\mathrm{l}+3 / 2
$$

which gives the energy eigenvalues as

$$
\mathrm{E}_{\mathrm{nl}}=\varepsilon \hbar \omega=\hbar \omega(2 \mathrm{n}+1+3 / 2)
$$

and corresponding radial wavefunction is then written in the following form by substituting equation (12) into equation (10)

$$
\mathrm{R}(\mathrm{r})=\mathrm{C}_{\mathrm{nl}} \mathrm{e}^{-\xi^{2} / 2} \xi^{\mathrm{l}+1} \mathrm{~F}\left(-\mathrm{n}, \mathrm{l}+3 / 2, \xi^{2}\right)
$$

where $\mathrm{C}_{\mathrm{nl}}$ is the normalization constant.

In order to evaluate the energy eigen values of a harmonic oscillator group theoretically we shall work with the raising and lowering operators for the eigenvalue of H defined by

$$
\begin{aligned}
& a_{j}=\frac{1}{(2 \mu \hbar \omega)^{1 / 2}}\left(P_{j}-i \omega \mu r_{j}\right) ; \\
& a_{j}^{+}=\frac{1}{(2 \mu \hbar \omega)^{1 / 2}}\left(P_{j}+i \omega \mu r_{j}\right) ;
\end{aligned}
$$

Using the commutation relations between $\mathrm{a}_{\mathrm{i}}$ and $\mathrm{a}_{\mathrm{j}}{ }^{+}$can be found to be 


$$
\begin{aligned}
& {\left[\mathrm{a}_{\mathrm{i}}, \mathrm{a}_{\mathrm{j}}^{+}\right]=\delta_{\mathrm{ij}}} \\
& {\left[\mathrm{a}_{\mathrm{i}}, \mathrm{a}_{\mathrm{j}}\right]=\left[\mathrm{a}_{\mathrm{i}}{ }^{+}, \mathrm{a}_{\mathrm{j}}^{+}\right]=0}
\end{aligned}
$$

investing the transformation (17) and substituting in to equation (1) the Hamiltonian of the system becomes

$$
\mathrm{H}=\hbar \omega \sum_{\mathrm{j}}\left(\mathrm{a}_{\mathrm{j}}{ }^{+} \mathrm{a}_{\mathrm{i}}+\frac{1}{2}\right)=\frac{\hbar \omega}{2} \sum_{\mathrm{j}}\left\{\mathrm{a}_{\mathrm{j}}{ }^{+}, \mathrm{a}_{\mathrm{i}}\right\}
$$

where $\{\mathrm{A}, \mathrm{B}\}=\mathrm{AB}+\mathrm{BA}$ denotes the anticommutator of $\mathrm{A}$ and $\mathrm{B}$.

The commutators of the raising and the lowering operators with the Hamiltonian turn out to be

$$
\left[\mathrm{H}, \mathrm{a}_{\mathrm{i}}^{+}\right]=\hbar \omega \mathrm{a}_{\mathrm{i}}^{+},\left[\mathrm{H}, \mathrm{a}_{\mathrm{i}}\right]=-\hbar \omega \mathrm{a}_{\mathrm{i}}
$$

The occupation number operator $\mathrm{a}_{\mathrm{i}}+\mathrm{a}_{\mathrm{i}}$ has eigenvalues $\mathrm{n}$; where $\mathrm{n}$; can take any non-negative integral values. The eigenvalues of the Hamiltonian (19) are therefore

$$
\mathrm{E}_{\mathrm{n}}=\left(\mathrm{n}+\frac{3}{2}\right) \hbar \omega
$$

where

$$
\mathrm{n}=\mathrm{n}_{1}+\mathrm{n}_{2}+\mathrm{n}_{3} ; \mathrm{n}_{1}, \mathrm{n}_{2}, \mathrm{n}_{3}=0,1,2 \ldots \ldots \ldots
$$

The degeneracy of the level $E_{n}$ is then easily seen to be $(n+1)(n+2) / 2$. The angular momentum operator can be worked out by using inverse transformation of (17) and is found to be

$$
\mathrm{L}_{\mathrm{j}}=(\overrightarrow{\mathrm{r}} \times \overrightarrow{\mathrm{P}})_{\mathrm{j}}=\frac{\mathrm{i} \hbar}{2} \sum_{\mathrm{k}, \mathrm{l}=1}^{3} \epsilon_{\mathrm{jkl}}\left(\mathrm{a}_{\mathrm{k}} \mathrm{a}_{\mathrm{l}}^{+}-\mathrm{a}_{\mathrm{k}}{ }^{+} \mathrm{a}_{\mathrm{l}}\right)
$$

We can further show that operators of the form $a_{i}{ }^{+} a_{j}$ commute with the Hamiltonian. The operators $\mathrm{a}_{\mathrm{i}}{ }^{+} \mathrm{a}_{\mathrm{j}}$ has the effect of transferring a quantum from the $\mathrm{j}^{\text {th }}$ direction to the $\mathrm{i}^{\text {th }}$ - direction and hence leaves the total number of quanta unchanged. There are nine such operators and it can be shown that they generate the algebra of $U(3)$. We see from (19) that the operator for the total quantum number is given by 


$$
\sum_{\mathrm{i}=1}^{3} \mathrm{a}_{\mathrm{i}}{ }^{+} \mathrm{a}_{\mathrm{j}}=\frac{\mathrm{H}}{\hbar \omega}-\frac{3}{2}
$$

and hence it commutes with all the operators $\mathrm{a}_{\mathrm{i}}{ }^{+} \mathrm{a}_{\mathrm{j}}$. Eight other linear combinations of the operator $\mathrm{a}_{\mathrm{i}}{ }^{+} \mathrm{a}_{\mathrm{j}}$ can be constructed which generate the algebra of SU(3). There are

$$
\left.\begin{array}{c}
\lambda_{1}=a_{1}^{+} a_{2}+a_{2}^{+} a_{1}, \lambda_{2}=-i\left(a_{1}^{+} a_{2}-a_{2}^{+} a_{1}\right) \\
\lambda_{3}=\left(a_{1}^{+} a_{1}-a_{2}^{+} a_{2}\right), \lambda_{y}=a_{1}^{+} a_{3}+a_{3}^{+} a_{1} \\
\lambda_{5}=-i\left(a_{1}^{+} a_{3}-a_{3}^{+} a_{1}\right), \lambda_{6}=a_{2}^{+} a_{3}+a_{3}^{+} a_{2} \\
\lambda_{7}=-i\left(a_{2}^{+} a_{3}-a_{3}^{+} a_{2}\right), \lambda_{8}=\frac{1}{\sqrt{3}}\left(a_{1}^{+} a_{1}+a_{2}^{+} a_{2}-2 a_{3}^{+} a_{3}\right)
\end{array}\right\}
$$

These operator satisfy the following relation

$$
\left[\lambda_{\mathrm{j}}, \lambda_{\mathrm{k}}\right]=2 \mathrm{i} \sum_{\mathrm{l}} \mathrm{f}_{\mathrm{jkl}} \lambda_{\mathrm{l}}
$$

The dynamical symmetry group of a three dimensional harmonic oscillator of monopole is therefore $\mathrm{SU}(3)$.

\section{BOUND STATES OF MONOPOLES}

In order to understand the behavior of a monopole moving in the field of another monopole we start with the following Schrodinger equation

$$
\hat{\mathrm{H}} \psi=\mathrm{E} \psi
$$

where $\hat{\mathrm{H}}$ is the Hamiltonian given as

$$
\hat{\mathrm{H}}=\frac{\hat{\mathrm{P}}^{2}}{2 \mathrm{~m}} \mathrm{~V}(\mathrm{r})
$$

$\mathrm{V}(\mathrm{r})$ is the interaction potential in central field approximation given as 


$$
V(r)=-\frac{g^{2}}{r}
$$

Substituting equation (3.3) and (3.2) to equation (3.1) we get

$$
\left[\frac{\hat{\pi}^{2}}{2 \mathrm{~m}}+\{\mathrm{V}(\mathrm{r})-\mathrm{E}\}\right] \psi=0
$$

Assuming the wave function as a product of radial and angular wave function

$$
\psi=\mathrm{R}(\mathrm{r}) \mathrm{Y}_{\mathrm{l}}^{\mathrm{m}}(\theta, \phi)
$$

we obtain

$$
\mathrm{r}^{2}\left\{\frac{1}{\mathrm{rR}(\mathrm{r})} \frac{\mathrm{d}^{2}}{\mathrm{dr}^{2}}(\mathrm{rR})+2 \mathrm{~m}(\mathrm{E}-\mathrm{V})\right\}=-\frac{\Lambda \mathrm{Y}_{\mathrm{l}}^{\mathrm{m}}(\theta, \phi)}{\mathrm{Y}_{\mathrm{l}}^{\mathrm{m}}(\theta, \phi)}
$$

where

$$
\Lambda=\frac{1}{\operatorname{Sin} \theta} \frac{\partial}{\partial \theta}\left(\operatorname{Sin} \theta \frac{\partial}{\partial \theta}\right)+\frac{1}{\operatorname{Sin}^{2} \theta} \frac{\partial^{2}}{\partial \phi^{2}}=1(1+1)
$$

which on solving as hydrogen atom problem yields the energy eigenvalue of a monopolonium (i.e. bound state of two monopoles) system in the $n^{\text {th }}$ eigen state, as follows

$$
E_{n}=-\frac{m g^{4}}{n^{2}}
$$

where $\mathrm{m}$ is the reduced mass.

The distance between the two generalized charges in unified space may be written as

$$
\mathrm{a}_{\mathrm{o}}=\frac{2}{\mathrm{mg}^{2}}
$$

This $\mathrm{a}_{\mathrm{o}}$ is the Bohr radius for a monopolonium. This is very small in comparison to atomic Bohr radius as the value of magnetic charge is very large in comparison to electric charge $\mathrm{e}^{(9,10,11,12)}$. The corresponding eigenfunctions are related to the associated Legendre polynomials and the complete solution is 


$$
\psi_{\mathrm{nlm}}(\mathrm{r})=\mathrm{R}_{\mathrm{nl}}(\mathrm{r}) \mathrm{Y}_{1}^{\mathrm{m}}(\theta, \phi)
$$

with first few $\mathrm{R}_{\mathrm{nl}}$ are given by

$$
\left.\begin{array}{c}
\mathrm{R}_{10}(\mathrm{r})=2\left(\frac{1}{\mathrm{a}_{\mathrm{o}}}\right)^{3 / 2} \exp \left(-\frac{\mathrm{r}}{\mathrm{a}_{\mathrm{o}}}\right) \\
\mathrm{R}_{20}(\mathrm{r})=\left(\frac{1}{2 \mathrm{a}_{\mathrm{o}}}\right)^{3 / 2}\left(2-\frac{\mathrm{r}}{\mathrm{a}_{\mathrm{o}}}\right) \exp \left(-\frac{\mathrm{r}}{2 \mathrm{a}_{\mathrm{o}}}\right) \\
\mathrm{R}_{21}(\mathrm{r})=\left(\frac{1}{2 \mathrm{a}_{\mathrm{o}}}\right)^{3 / 2} \frac{\mathrm{r}}{\mathrm{a}_{\mathrm{o}} \sqrt{3}} \exp \left(-\frac{\mathrm{r}}{2 \mathrm{a}_{\mathrm{o}}}\right)
\end{array}\right\}
$$

The normalization constants are such that

$$
\int_{0}^{\infty}\left|R_{n}(r)\right|^{2} r^{2} d r=1
$$

For the ground state, the Spherically symmetric wave function is given by

$$
\psi_{100}=\frac{1}{\sqrt{4 \pi}}\left(\frac{1}{a_{0}}\right)^{3 / 2} 2 \exp \left(-\frac{r}{a_{0}}\right)
$$

and the probability of finding two dyons at a distance $r$ apart, is given by

$$
\begin{aligned}
& P(r)=\frac{4 r^{2}}{a_{0}^{3}} \exp \left(-\frac{2 r}{a_{0}}\right) \\
& \int_{0}^{\infty} P(r) d r=1
\end{aligned}
$$

which shows part that $\mathrm{P}(\mathrm{r})$ is zero at $\mathrm{r}=\mathrm{o}$ and $\mathrm{r}=\infty$ and has a maximum value at $\mathrm{r}=\mathrm{a}_{0}$. To analyze this system group theoretically we define the following Runge-lenz vector,

$$
M^{\prime}=\frac{1}{2 \mu}(\vec{P} \times \vec{L}-\vec{L} \times \vec{P})-q^{2} \frac{\vec{r}}{r}
$$


which is a constant of motion i.e. it commutes with the Hamiltotian (1). $\hat{\mathrm{L}}$ is the orbital angular momentum in equation (40) this angular momentum operator which is normal to the plane of orbit commute with Runge-Lenz vector i.e.

$$
M^{\prime} . \mathrm{L}=0
$$

so that $\mathrm{M}^{\prime}$ is a vector in the plane of the orbit. The orbital angular momentum also commutes with the Hamiltonian and is a constant of motion. We thus have

$$
\left[\mathrm{M}^{\prime}, \mathrm{H}\right]=0,[\mathrm{~L}, \mathrm{H}]=0
$$

We get from equation (40) with the help of these commutation relations

$$
\overrightarrow{\mathrm{M}}^{\prime 2}=\frac{2 \mathrm{H}}{\mu}\left(\overrightarrow{\mathrm{L}}^{2}+\hbar^{2}\right)+\mathrm{z}^{2} \mathrm{~g}^{2}
$$

We now have six operators which correspond to the invariants of the problem at hand an can be used to generate unitary transformations under which the Hamiltonian would be invariant. There will be fifteen commutators which are given below in five equations each standing for three equations obtainable from it by cyclic permutation of $\mathrm{x}, \mathrm{y}$, and $\mathrm{z}$ :

$$
\left.\begin{array}{c}
{\left[\mathrm{L}_{\mathrm{x}}, \mathrm{L}_{\mathrm{y}}\right]=\mathrm{i} \hbar \mathrm{L}_{\mathrm{z}},\left[\mathrm{M}_{\mathrm{x}}^{\prime}, \mathrm{L}_{\mathrm{x}}\right]=0,\left[\mathrm{M}_{\mathrm{x}}^{\prime}, \mathrm{L}_{\mathrm{y}}\right]=\mathrm{i} \hbar \mathrm{M}_{\mathrm{z}}^{\prime}} \\
{\left[\mathrm{M}_{\mathrm{x}}^{\prime}, \mathrm{L}_{\mathrm{z}}\right]=-\mathrm{i} \hbar \mathrm{M}_{\mathrm{y}}^{\prime},\left[\mathrm{M}_{\mathrm{x}}^{\prime}, \mathrm{M}_{\mathrm{y}}^{\prime}\right]=-\frac{2 \mathrm{i} \hbar}{\mu} \mathrm{HL}_{\mathrm{z}}}
\end{array}\right\}
$$

The components of $\mathrm{L}$ themselves constitute a closed algebra can be used to generate the lie group $\mathrm{O}(3)$. Let us restrict ourselves to a particulars bound state energy level $(\mathrm{E}<0)$ of the monopolonium and confine ourselves to the invariant subspace ( of the full space) which corresponds to the eigenvalue $\mathrm{E}$. In this subspace, we can replace $\mathrm{H}$ by $\mathrm{E}$ and define a new operator by

$$
\overrightarrow{\mathrm{M}}=(-\mu / 2 \mathrm{E})^{1 / 2} \overrightarrow{\mathrm{M}}^{\prime}
$$

In the first commutators of (44) the components of $\vec{M}$ simply replace those of $\overrightarrow{\mathrm{M}}^{\prime}$. The last commutator, however, takes the form

$$
\left[\mathrm{M}_{\mathrm{x}}, \mathrm{M}_{\mathrm{y}}\right]=\mathrm{i} \hbar \mathrm{L}_{\mathrm{z}}
$$


Now these can be used to generate a six parameter Lie group which will be the dynamical symmetry of the monopolonium.

To show that this group is $\mathrm{O}(4)$, we define six operators by writing

$$
J_{i j}=\sum_{k} \epsilon_{i j k} L_{k} \text { for } i, j, k=x, y, z
$$

and

$$
\mathrm{J}_{\mathrm{i} \omega}=-\mathrm{J}_{\omega \mathrm{i}}=\mathrm{M}_{\mathrm{i}}
$$

Here, $\epsilon_{\mathrm{ijk}}$ is the fully antisymmetric tensor of rank three. The commutation relations between these operators can be worked out and are found to be

$$
\left.\begin{array}{c}
{\left[\mathrm{J}_{\mathrm{xy}}, \mathrm{J}_{\mathrm{yz}}\right]=\mathrm{i} \hbar \mathrm{J}_{\mathrm{zx}},\left[\mathrm{J}_{\mathrm{x} \omega}, \mathrm{J}_{\mathrm{yz}}\right]=0} \\
{\left[\mathrm{~J}_{\mathrm{x} \omega}, \mathrm{J}_{\mathrm{zx}}\right]=\mathrm{i} \hbar \mathrm{J}_{\mathrm{z} \omega},\left[\mathrm{J}_{\mathrm{x} \omega}, \mathrm{J}_{\mathrm{y} \omega}\right]=\mathrm{i} \hbar \mathrm{J}_{\mathrm{xy}}} \\
{\left[\mathrm{J}_{\mathrm{x} \omega}, \mathrm{J}_{\mathrm{yx}}\right]=\mathrm{i} \hbar \mathrm{J}_{\mathrm{y} \omega}}
\end{array}\right\}
$$

where, again, each equation stands for three equations obtained from it by cyclic permutation of $x, y$, and $z$. The six operators $J_{\rho \sigma}(\rho, \sigma=x, y, z, \omega)$ are infinitesimal generators of a group whose operation leaves the quadratic form $x^{2}+y^{2}+z^{2}+\omega^{2}$ invariant i.e. the group of all real orthogonal transformations in a four-dimensional vector space or $\mathrm{O}(4)$. We have one operator each for generating rotations in the six coordinate plane.

Let us construct the following Linear combination of $\overrightarrow{\mathrm{L}}$ and $\overrightarrow{\mathrm{M}}$

$$
\overrightarrow{\mathrm{A}}=\frac{1}{2}(\overrightarrow{\mathrm{L}}+\overrightarrow{\mathrm{M}}) ; \quad \overrightarrow{\mathrm{B}}=\frac{1}{2}(\overrightarrow{\mathrm{L}}-\overrightarrow{\mathrm{M}})
$$

so that commutation relations between the components of $\vec{A}$ and $\vec{B}$ are

$$
\begin{aligned}
& \vec{A} \times \vec{A}=i \hbar \vec{A}, \vec{B} \times \vec{B}=i \hbar \vec{B} \\
& {\left[A_{i}, B_{j}\right]=0 \text { for } i, j=x, y, z}
\end{aligned}
$$

Moreover, since $\overrightarrow{\mathrm{L}}$ and $\overrightarrow{\mathrm{M}}$ both commute with $\mathrm{H}$, it follows that $\overrightarrow{\mathrm{A}}$ and $\overrightarrow{\mathrm{B}}$ also do. The above equation shows that the Lie algebra of $\vec{A}$ and $\vec{B}$ are separately closed, so that each of them can be used to generate the $\mathrm{SU}(2)$ group. This tells that $\mathrm{O}(4)$ is homomorphic to $\mathrm{SU}(2) \times \mathrm{SU}(2)$.

We may choose the two commutating generator to be any one component of $\vec{A}$ and any one component of $\vec{B}$. There are therefore two Casimir operators which commute with all the six generators. There are obviously $\overrightarrow{\mathrm{A}}^{2}$ and $\overrightarrow{\mathrm{B}}^{2}$ or any two 
independent linear combination of these. Their eigenvalues in analogy with the theory of $\mathrm{SU}(2)$ may be written as

$$
\overrightarrow{\mathrm{A}}^{2}=\mathrm{a}(\mathrm{a}+1) \hbar^{2} ; \quad \overrightarrow{\mathrm{B}}^{2}=\mathrm{b}(\mathrm{b}+1) \hbar^{2}
$$

where a and $b$ take all non-negative integral or half odd integral values. Taking the sum and the difference of $\overrightarrow{\mathrm{A}}^{2}$ and $\overrightarrow{\mathrm{B}}^{2}$, we find that

$$
\mathrm{C} \equiv \overrightarrow{\mathrm{A}}^{2}+\overrightarrow{\mathrm{B}}^{2} \frac{1}{2}\left(\mathrm{~L}^{2}+\mathrm{M}^{2}\right), \quad \mathrm{C} \equiv \overrightarrow{\mathrm{A}}^{2}-\overrightarrow{\mathrm{B}}^{2}=\overrightarrow{\mathrm{L}} \cdot \overrightarrow{\mathrm{M}}
$$

Using (41) and (45) the second of the above equation shows that $C^{\prime}=0$ that our physical system (i.e. monopolonium) corresponds only to that part of $\mathrm{O}(4)$ for which $\overrightarrow{\mathrm{A}}^{2}=\overrightarrow{\mathrm{B}}^{2}$ or $\mathrm{a}(\mathrm{a}+1)=\mathrm{b}(\mathrm{b}+1)$. This gives the two solutions $\mathrm{a}=\mathrm{b}$ and $\mathrm{a}=-(\mathrm{b}+1)$; the second solution must, however, be discarded since $a$ and $b$ are restricted to non-negative values. This tells us that only those representation of $\mathrm{O}(4)$ represents the state of monopolonium for which $\mathrm{a}=\mathrm{b}$ i.e. representation of the form $(\mathrm{a}, \mathrm{a})$.

The eigenvalues of Casimir operator $C$ then becomes

$$
\mathrm{C}=2 \mathrm{a}(\mathrm{a}+1) \hbar^{2}
$$

Using (43), (45) and (53), we than have that

$$
\begin{aligned}
C & =\frac{1}{2}\left[\overrightarrow{\mathrm{L}}^{2}-\frac{\mu}{2 \mathrm{E}}\left\{\frac{2 \mathrm{E}}{\mu}\right\}\left(\mathrm{L}^{2}+\hbar^{2}\right)+\mathrm{g}^{2}\right] \\
& =-\frac{1}{2}\left[\hbar^{2}-\frac{\mu \mathrm{g}^{4}}{2 \mathrm{E}}\right]
\end{aligned}
$$

Using (51) in the above equation, this finally gives

$$
\mathrm{E}=-\frac{\mu \mathrm{g}^{4}}{2 \hbar^{2}(2 \mathrm{a}+1)^{2}}
$$

where $\mu$ is the reduced mass.

If we make identification $n=2 a+1$, so that $n$ takes all positive integral values (52) agrees with energy eigenvalues the energy eigen values of monopolnium as obtained in the previous section. Since the dimension of the irreducible representation $(\mathrm{a}, \mathrm{a})$ of $\mathrm{O}(4)$ is $(2 \mathrm{a}+1)^{2}=\mathrm{n}^{2}$ this also explains the $\mathrm{n}^{2}$ fold degeneracy of the levels.

\section{CONCLUSION}

Equation (1) is the Hamiltonian operator for harmonic oscillator and equation (3) is the Schrodinger equation for this oscillator. Solving this equation we obtained the energy eigenvalue for this system given by equation (15) and equation (16) is the radial wave function for this system. Equation (17) describe the raising and lowering operator 
for this system which leads to Hamiltonian of the system given by equation (19). The energy eigenvalue of the system are given by equation (21) which are obtained group theoretically. The degeneracy of the system is $(n+1)(n+2) / 2$. Equation $(23)$ is the angular momentum operator for this system. Equation (24) and (25) are the operators which generate the algebra of $\mathrm{SU}(3)$. Equation (26) is the relation satisfied by these operator showing that dynamical symmetry group of a three dimensional harmonic oscillator is $\mathrm{SU}(3)$.

Equation (27) is the Schrodinger equation describing the motion of a monopole moving in the field of another monopole in central field approximation. This equation yields the energy eigen value for monopolonium given by equation (32). Equation (33) is the Bohr radius of this system which is very small in comparison to atomic Bohr radius and energy of this system is very high because of magnetic charge. Equation (35) gives the radial wave function of the system. Equation (40) is the Runge-Lenz vector for the system. Casimir operator for the system is given by equation (53). The energy eigen value of the system obtained group theoretically are given by equation (54) this also explains the $\mathrm{n}^{2}$ fold degeneracy of the levels.

\section{ACKNOWLEDGMENT}

One of the author S. C. Joshi is thankful to DSA/COSIST, New Delhi for providing financial assistant.

\section{REFERENCES}

1. P.A.M Dirac, Proc. Roy. Sco. london A $\underline{133}$,60 (1931).

2. P.A.M Dirac, Phys. Rev. $\underline{74}, 817$ (1948).

3. H.J. Lipkin, W.I. Weisberger and M. Peshkin, Ann. Phys. ( N.Y ) 53 ,203 (1969).

4. J. Schwinger, Phys. Rev. 144,1087 (1966).

5. D. Zwanzigar, Phys. Rev. D3 , 880 (1971), Phys. Rev. 176 , 1480 (1968).

6. P.P. Purohit, V.P. Pandey and B.S. Rajput, Ind. J. of Pure \& Appl. Phys. 37 , 163 (1999).

7. P.C. Pant, V.P. Pandey and B.S. Rajput, I1 Nuovo Cimento (In press).

8. P.P. Purohit, V.P. Pandey and B.S. Rajput, I1 Nuovo Cimento (communicated).

9. D. Akers, IL Nuovo Cim. A $\underline{105}$, 935 (1992).

10. D. Akers, Int. J. Theor. Phys. 26 , 613 (1987).

11. P.P. Purohit, V.P. Pandey and B.S. Rajput, Ind. J. of Pure \& Appl. Phys. (In press)

12. P.P. Purohit, V.P. Pandey and B.S. Rajput, Int. J. of Mod. Phys. A (communicated). 